Revisão / Review

\title{
Sistema de grupo sangüíneo Duffy: Biologia e prática transfusional
}

\section{Duffy blood group system: Biology and transfusion practice}

Eduardo Jens ${ }^{l}$

Thiago Pagliarini ${ }^{1}$

Marcia C. Z. Novaretti ${ }^{2}$

\begin{abstract}
Após a introdução da técnica de antiglobulina indireta por Coombs em meados da década de 40, vários anticorpos antieritrocitários foram descobertos. O grupo sanguíneo Duffy foi descoberto quando Cutbush e Ikin detectaram, no início da década de 50, os primeiros anticorpos desse sistema. Os anticorpos Duffy são clinicamente significantes na prática transfusional, pois mostraram ser causadores de reação hemolítica transfusional e de doença hemolítica do recém-nascido, sendo de ocorrência mundial. O gene FY é constituído por dois exons e seu lócus foi mapeado no cromossomo 1q22-q23. Os antígenos $F y^{a}$ e $F_{y^{b}}$ são codificados pelos alelos FYA e FYB e são responsáveis pelos fenótipos $F y(a+b-), F y(a-b+)$ e $F y(a+b+)$. São carreados por uma glicoproteina de 336 aminoácidos também chamada DARC (Duffy Antigen/Receptor for Chemokines), que tem alta afinidade a quimiocinas, sendo também os receptores para Plasmodium vivax. Os polimorfismos relacionados aos seus alelos permitiram o desenvolvimento da técnica de genotipagem por PCR, que é de grande utilidade para a segurança transfusional e incompatibilidade feto-materna. Na última década, inúmeras pesquisas têm sido feitas quanto ao papel biológico dos antígenos de grupos sangüíneos. Nesse artigo iremos revisar o sistema de grupo sangüineo Duffy, em especial quanto à prática transfusional e suas funções biológicas. Rev. bras. hematol. hemoter. 2005;27(2):110-119.
\end{abstract}

Palavras-chave: Sistema de grupo sangüineo Duffy; DARC; quimiocinas; malária; gene Duffy.

\section{Introdução}

Um dos mais notáveis avanços em pesquisa na área médica na primeira metade do século XX foi a identificação de antígenos eritrocitários e o reconhecimento de sua importância na prática transfusional e na doença hemolítica do recém nascido. ${ }^{1,2}$

Mais recentemente, houve grandes progressos no entendimento e análise estrutural e funcional dos antígenos de grupos sangüíneos expressos tanto nas hemácias quanto em tecidos não eritróides. ${ }^{3}$ Neste artigo iremos revisar o sistema grupo sangüíneo Duffy, que tem sido alvo de pesquisas, pelo particular interesse fisiológico e transfusional a ele relacionado, sendo considerado um dos mais interessantes loci cromossômicos para avaliar o impacto da pressão da seleção natural em diferentes regiões geográficas. ${ }^{4,5}$

A descoberta do anti-Fy ${ }^{a}$ ocorreu em 1950, por Cutbush et al, ${ }^{6}$ que detectaram uma aglutinina no soro de um paciente hemofílico politransfundido ainda não reconhecida como antígeno de grupo sangüíneo. Esse anticorpo

\footnotetext{
${ }_{2}^{I}$ Pós-graduando da Disciplina de Hematologia da Faculdade de Medicina da Universidade de São Paulo.

Professora colaboradora da Disciplina de Hematologia da Faculdade de Medicina da Universidade de São Paulo. Chefe da Divisão de Imunematologia da Fundação Pró-Sangue Hemocentro de São Paulo.
}

Divisão de Imunematologia da Fundação Pró-Sangue Hemocentro de São Paulo/Disciplina de Hematologia da Faculdade de Medicina da Universidade de São Paulo.

Correspondência para: Marcia Cristina Zago Novaretti

Av. Dr. Enéas de Carvalho Aguiar, $155-1^{\circ}$ andar

05403-000 - São Paulo-SP - Brasil

Tel: 5511 3088-2638; Fax.: 5511 3083-2290

E-mail:marcitabrz@yahoo.com 
foi chamado anti-Fy, em homenagem ao paciente em questão, Sr. Duffy, e reagia com 64,9\% das 205 amostras de sangue testadas de indivíduos não aparentados na população inglesa. $^{7}$

No ano seguinte, Ikin et $\mathrm{al}^{8}$ descreveram o anti-Fy ${ }^{\mathrm{b}}$, anticorpo que define o par antitético do antígeno Fya. Em 1955, Sanger et al ${ }^{9}$ observaram que o fenótipo Fy(a-b-) era o mais comum em afro-americanos e que provavelmente representava um produto de um alelo silencioso, $F Y$.

Chown et a $1^{10}$ descreveram outro alelo no locus DUFFY, $F Y X$, co-dominante com $F Y A$ e recessivo para $F Y B$, correlacionado com a expressão fraca do antígeno $\mathrm{Fy}^{\mathrm{b}}$. $\mathrm{O}$ alelo $F Y X$ foi posteriormente descrito com mais detalhe por Lewis et al, ${ }^{11}$ que demonstraram que o gene FY não produz um antígeno diferente dos outros do sistema Duffy, mas os eritrócitos reagem mais fracamente com soros anti-Fyb, podendo ser detectado por métodos de adsorção e eluição.

Os anti-soros anti-Fy ${ }^{\mathrm{a}} \mathrm{e}$ anti-Fy $\mathrm{y}^{\mathrm{b}}$ definem os fenótipos Fy(a+b-), Fy(a+b+), Fy(a-b+) e Fy(a-b-). Em 1971, o antígeno Fy3 foi descrito e os antígenos Fy4 e Fy5 foram reportados em 1973. ${ }^{12,13,14}$ Os anti-soros que definem os fenótipos Fy3 e Fy5 são muito raros e não existem comercialmente.

Em 1987, o primeiro anticorpo monoclonal murino anti-Fy6 foi obtido e definiu outro determinante antigênico Duffy (Fy6) presente em todas as células Duffy-positivo mas ausente em células Fy (a-b-). Esse epítopo se revelou importante pelos estudos de estrutura-função do sistema Duffy. ${ }^{15}$

Um dos aspectos interessantes dos antígenos Duffy é sua função de receptor de merozoítas de Plasmodium vivax e de $P$. knowlesi, que são respectivamente os agentes responsáveis por diferentes formas de malária no homem e no macaco. Essa função foi colocada em evidência por Miller et al, ${ }^{16} \mathrm{em}$ 1975, mostrando a resistência dos eritrócitos Fy (a-b-) à invasão de merozoítas de $P$. knowlesi que eram cultiváveis in vitro naquela época. Em 1989, Barnwell et al ${ }^{17}$ confirmaram os mesmos experimentos com P.vivax.

A partir de 1989, os estudos referentes ao sistema Duffy tomaram um novo impulso. A purificação da proteína Duffy e a clonagem do cDNA permitiram a abordagem de estudos estruturais e funcionais. ${ }^{18,19} \mathrm{~A}$ homologia dessa proteína com os receptores de quimiocinas, peptídeos implicados na resposta inflamatória, nos levou ao estudo da função biológica do grupo sangüíneo Duffy. ${ }^{20,21}$

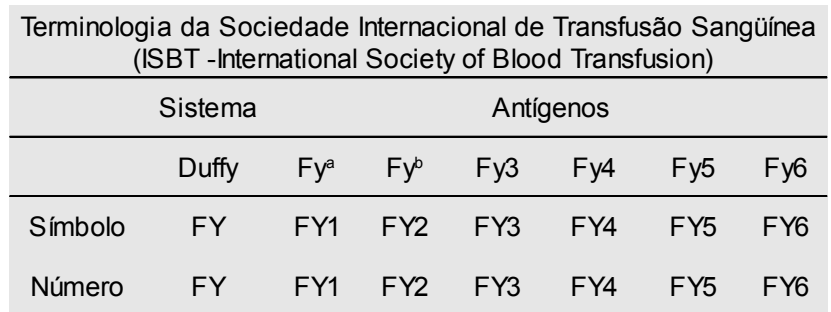

\section{Antígenos}

Os antígenos $\mathrm{Fy}^{\mathrm{a}}$ e $\mathrm{Fy}^{\mathrm{b}}$ são codificados por duas formas alélicas do gene $F Y$. Os alelos $F Y A$ e $F Y B$ diferem por uma simples substituição de base no nucleotídeo 125 . No alelo $F Y A$, a base é guanina (G) e no alelo $F Y B$ a base é adenina (A). Isso produz um códon para glicina no aminoácido 42 no alelo $F Y A$, e um códon para ácido aspártico no alelo $F Y B$. Essa substituição de um aminoácido no domínio amino-terminal da proteína é suficiente para definir os dois antígenos antitéticos. ${ }^{23,24,25}$ Essa variação leva à identificação dos fenótipos Fy(a+b-), Fy(a-b+) e Fy $(a+b+) .{ }^{26}$

Estudos com anticorpos monoclonais realizados por Wasniowska et $\mathrm{al}^{27}$ mostraram que o sítio de maior imunogenicidade para os antígenos $\mathrm{Fy}^{\mathrm{a}} / \mathrm{Fy}^{\mathrm{b}}$ está localizado entre os aminoácidos 37 e 47.

Tournamille et al, ${ }^{28}$ estudando quatro amostras de doadores de plaquetas com fenótipo $\mathrm{Fy}\left(\mathrm{a}+\mathrm{b}+{ }^{+ \text {fraco }}\right)$, descobriram que a alteração da expressão do alelo $F Y B$ é devida à mutação específica no nucleotídeo $265 \mathrm{C}>\mathrm{T}$ do alelo $F Y X$, responsável pela expressão muito baixa do fenótipo $\mathrm{Fy}^{\mathrm{b}}$. Essa substituição de base provoca uma mudança do aminoácido Arginina por uma Cisteína na posição 89 do gene $F Y$.

Técnicas de adsorção e eluição são utilizadas para detectar a presença do fenótipo Fy ${ }^{\mathrm{b}}$ fraco mas nem sempre essas técnicas são eficazes.

Em um estudo com cinco amostras de indivíduos com fenótipo $\mathrm{Fy}\left(\mathrm{a}+\mathrm{b}+{ }^{\text {fraco }}\right)$ e duas amostras $\mathrm{Fy}\left(\mathrm{a}-\mathrm{b}+{ }^{\text {fraco }}\right)$ de indivíduos não aparentados, Olsson et al, ${ }^{29}$ além da mutação $265 \mathrm{C}>\mathrm{T}$, observaram em todas as amostras pesquisadas a mutação $298 \mathrm{G}>\mathrm{A}$, também associada ao alelo $F Y X$. Essa mutação provoca uma substituição de Alanina por Treonina no aminoácido 100 do gene $F Y$.

Outros autores demonstraram sorologicamente que os antígenos Fy3 e Fy5 também têm suas expressões enfraquecidas em eritrócitos. ${ }^{30,31,32}$

Castilho et $\mathrm{al}^{33}$ descreveram um novo polimorfismo de um único nucleotídeo (SNP - single nucleotide polymorphism) $145 \mathrm{G}>\mathrm{T}$ no alelo $F Y B$, que foi encontrado juntamente com os SNPs dos nucleotídeos 265 e 298, em dois doadores de sangue brasileiros não aparentados, um caucasiano e outro negro. Nesse estudo foi observado que, dos 361 doadores estudados, em 27 (7,5\%) encontrou-se apenas a mutação 298 $\mathrm{G}>\mathrm{A}$, mas nenhum dos indivíduos estudados apresentou a mutação $265 \mathrm{C}>\mathrm{T}$ isoladamente.

$\mathrm{O}$ fenótipo Fy(a-b-) em negros mostrou ser devido a uma mutação pontual $-33 \mathrm{~T}>\mathrm{C}$ na região promotora do gene FYB, o GATA-box. ${ }^{34,35}$ Essa alteração leva a uma interrupção no fator de transcrição eritróide GATA-1, resultando na ausência de expressão do antígeno $\mathrm{Fy}^{\mathrm{b}}$ apenas no eritrócito, não alterando a expressão dessa proteína em outros tecidos. Conseqüentemente, esses indivíduos podem vir a desenvolver anti-Fy ${ }^{\mathrm{a}}$, mas não anti-Fy ${ }^{\mathrm{b}}{ }^{36}$ 
Castilho et $\mathrm{a}^{33}$ observaram uma alta freqüência da mutação $-33 \mathrm{~T}>\mathrm{C}(12,5 \%)$ em indivíduos com ancestrais caucasianos, confirmando que na população brasileira não existe uma clara distinção de raças. Foi observada também a ocorrência simultânea das mutações $-33 \mathrm{~T}>\mathrm{C}, 265 \mathrm{C}>\mathrm{T}$ e $298 \mathrm{G}>\mathrm{A}$ $(1,7 \%)$ e a ocorrência das mutações $265 \mathrm{C}>\mathrm{T}$ e $298 \mathrm{G}>\mathrm{A}$ em indivíduos de descendência africana, o que difere de outros relatos na literatura que apresentaram a ocorrência das mutações 265 C > T e 298 G>A em 2-5\% da população caucasiana mas não em negros. ${ }^{29,37,38}$

O resultado de eventos naturais na região GATA-box para o alelo $F Y A^{\text {nulo }}$ é interessantemente encontrado em regiões endêmicas do Plasmodium vivax na Nova Guiné ${ }^{39} \mathrm{e}$, recentemente, na região amazônica. ${ }^{40}$

Shimizu et $\mathrm{al}^{41,42}$ investigaram várias etnias de regiões da Tailândia e Indonésia, verificando a alta incidência do alelo $F Y A(>0,9)$ e a presença do novo fenótipo $F y(a+$ fraco $b-)$, dados similares aos encontrados em estudos fenotípicos anteriores realizados na região do sudeste asiático e Oceania. Chama a atenção que os indivíduos Fy $\left(\mathrm{a}+{ }^{\text {fraco }} \mathrm{b}\right.$ - $)$ apresentaram genotipagem $F Y A / F Y A$ e os Fy(a-b-) apresentaram genotipagem $F Y A / F Y A$ ou $F Y A / F Y B$, por PCR-RFLP utilizando as enzimas BanI e Sty, porém os mecanismos genéticos que causam esse fenótipo não foram determinados. As implicações da presença desses fenótipos encontrados ainda são desconhecidas, mas sugerem que, em regiões endêmicas de malária, também há mecanismos de defesa para a infecção do $P$. vivax, distintos dos encontrados em descendentes africanos. ${ }^{42}$

Rios et a ${ }^{44}$ relataram a análise de três caucasóides que apresentavam fenótipos Fy(a-b-) nos quais foram identificados anti-Fy 3 em altos títulos.

No primeiro caso, a genotipagem apresentava-se $F Y A$ / $F Y A$ e as mutações $265 \mathrm{C}>\mathrm{T}, 298 \mathrm{G}>\mathrm{Ae}-33 \mathrm{~T}>\mathrm{C}$ não foram encontradas, porém uma mutação pontual $287 \mathrm{G}>\mathrm{A}$ foi observada, provocando a substituição de um nucleotídeo, acarretando um códon de término ("stop" códon). O segundo paciente apresentou genotipagem $F Y B / F Y B$ e as mutações $265 \mathrm{C}>\mathrm{T}$, $298 \mathrm{G}>$ A e $-33 \mathrm{~T}>\mathrm{C}$ não foram encontradas, mas a seqüência de nucleotídeos do exon 2 do gene $F Y$ mostrou uma mutação $407 \mathrm{G}>\mathrm{A}$ que causa um códon de término prematuro no aminoácido de posição 136. O terceiro paciente, previamente estudado por Mallinson et al, ${ }^{24}$ apresentava genótipo $F Y A$ / $F Y A$. As mutações $265 \mathrm{C}>\mathrm{T}, 298 \mathrm{G}>\mathrm{A}$ e $-33 \mathrm{~T}>\mathrm{C}$ não foram encontradas e a seqüência de nucleotídeos do exon 2 do $F Y$ mostrou uma mutação $408 \mathrm{G}>$ A que causa um códon de término prematuro no aminoácido de posição 136. Segundo os autores, essas mutações mostraram ser eventos espontâneos pois foram observadas no alelo $F Y A$ e no alelo $F Y B$.

$\mathrm{O}$ antígeno Fy3 foi descrito por Albrey et al, ${ }^{12}$ é resistente aos tratamentos com tripsina, DTT $0,2 \mathrm{M}, \alpha$-quimiotripsina, papaína, sialidase e pronase. ${ }^{45} \mathrm{O}$ epítopo conformacional Fy3 está localizado na terceira alça extracelular da glicoproteína Duffy, e seus determinantes antigênicos foram demonstrados por ensaios com anticorpos monoclonais serem entre os aminoácidos 281 e 285. ${ }^{27,46}$

Outros epítopos relativos à glicoproteína Duffy foram definidos por raros soros humanos, como os antígenos Fy4 e Fy5. ${ }^{27,46}$

O antígeno Fy4 foi definido por Behzad et a ${ }^{13} \mathrm{em}$ 1973, relatando um anticorpo que reagia somente com hemácias Fy(a-b-) de indivíduos com descendência africana. No mesmo ano, Colledge et al ${ }^{14}$ relataram o anti-Fy5, definindo o antígeno Fy5 como tendo uma possível interação com as proteínas Rh e Duffy. A raridade desses anticorpos impediu o progresso na caracterização dos epítopos correspondentes.

O antígeno Fy6, reconhecido por anticorpos monoclonais de classe IgG1 Kappa, foi mapeado na alça aminoterminal da proteína Duffy. É resistente a tratamentos com tripsina, DTT 0,2M e sialidase e sensível ao tratamento com papaína, $\alpha$-quimiotripsina e pronase. ${ }^{15,47,48}$ Relacionado com a suscetibilidade à invasão do $P$. vivax, Fy6 está presente nas hemácias, com exceção das Fy(a-b-), numa freqüência semelhante à do Fy3. ${ }^{15}$

Estudos com anticorpos monoclonais anti-Fy6 mostraram que a região imunodominante do epítopo linear Fy6 é o heptapeptídeo QLDFEDV na posição 19-25 da glicoproteína Duffy.

Utilizando técnica de citometria de fluxo, Woolley et $\mathrm{al}^{49}$ descreveram que a expressão de antígenos Duffy era maior em reticulócitos do que em eritrócitos maduros. A avaliação da expressão dos antígenos Duffy nos reticulócitos pode ser de valia no entendimento de como essa expressão é modulada em respostas inflamatórias agudas como cirurgias ou sepsis.

\section{Freqüência}

Em um estudo realizado por Novaretti et a ${ }^{50}$ em doadores de sangue na cidade de São Paulo, a freqüência fenotípica encontrada, para o sistema de grupo sangüíneo Duffy, foi de 19,8\% em caucasóides e 14\% em negros para o fenótipo Fy(a+b-). Quanto ao fenótipo Fy $(a+b+)$, a casuística foi de $41,4 \%$ para caucasóides e 1,6\% para negros. O fenótipo Fy(ab+) observado foi de 37,8\% para caucasóides e 17,5\% para negros. Em caucasóides, a freqüência do fenótipo Fy(a-b-) foi de $1,1 \%$, enquanto para os negros foi de $66,9 \%$, sendo esse fenótipo considerado um marcador de raça negra.

A grande diversidade de distribuição dos determinantes antigênicos Duffy, nos diversos grupos étnicos, é característica desse sistema de grupo sangüíneo.

Os determinantes antigênicos Fy ${ }^{\mathrm{a}}$ são prevalentes entre chineses, japoneses e melanésios, porém apresenta baixa freqüência entre negros africanos..$^{41,42}$ No entanto, o antígeno Fy é mais abundante na população caucasóide do que em asiáticos e negros africanos e americanos e o fenótipo Fy(ab-) é extremamente raro fora da população negra. ${ }^{16,51}$ 
Sandler et al,$^{52}$ investigando a origem da hemoglobina $\mathrm{S}$ em dois grupos nativos de sicilianos brancos, encontraram o fenótipo Fy(a-b-) em 11\% de indivíduos não aparentados, mostrando que a hemoglobina $\mathrm{S}$ é somente um dos genes africanos múltiplos atuais em populações brancas contemporâneas.

\section{Anticorpos}

Os anticorpos anti-Fy ${ }^{\mathrm{a}}$ e anti-Fy ${ }^{\mathrm{b}}$ podem causar reações transfusionais hemolíticas imediatas e tardias e também podem levar à doença hemolítica do recém-nascido..$^{53}$

Anti-Fy ${ }^{\mathrm{a}} \mathrm{e}$ anti-Fy $\mathrm{y}^{\mathrm{b}}$ ão predominantemente anticorpos de subclasse tipo IgG1. Em uma série de estudos, Szymanski et a ${ }^{54}$ verificaram que a maioria dos anticorpos anti-Duffy era composta de IgG1, 18\% eram IgG2 e $25 \%$ eram IgM.

$\mathrm{O}$ anti-Fy é mais freqüentemente produzido a partir de sensibilização por transfusão sangüínea que por gestação e não é de ocorrência natural. Sua freqüência é aproximadamente três vezes menor que o anti-K. Cerca de $50 \%$ dos anti$\mathrm{Fy}^{\mathrm{a}}$ ativam complemento acima do estágio $\mathrm{C} 3 .{ }^{55}$

Já o anti-Fy é cerca de vinte vezes menos comum que o anti-Fy ${ }^{\mathrm{a}}$ geralmente é encontrado em associação com outros anticorpos. Um exemplo de ocorrência natural de um potente anti-Fy foi descrito por Issit et al..$^{56}$ Também há relato de um caso de reação transfusional hemolítica devido à anti-Fy ${ }^{\mathrm{b}}$ causado por resposta imune primária. ${ }^{57}$

Os anticorpos Anti-Fy3 descrito por Albrey et $\mathrm{al}^{12} \mathrm{e}$ anti-Fy5 descrito por Colledge et $\mathrm{al}^{14}$ são anticorpos raros e reagem com todos os eritrócitos exceto em fenótipos Fy(a-b-). Esse último não reage também com eritrócitos $\mathrm{Rh}_{\text {null }} .53$

O anti-Fy3 pode causar reação transfusional hemolítica imediata e tardia e $o$ anti-Fy 5 pode causar reação transfusional hemolítica tardia e ambos não foram implicados em doença hemolítica do recém-nascido até o momento. ${ }^{53}$

O único exemplo de anti-Fy4 descrito em 1973, por Behzad et al, ${ }^{13}$ reagia com todos os eritrócitos com fenótipo Fy(a-b-) de negros e com a maioria dos fenótipos Fy $(a-b+)$ e $\mathrm{Fy}(\mathrm{a}+\mathrm{b}-)$ da mesma etnia. $\mathrm{O}$ anti-Fy4 não reagia com células $\mathrm{Fy}(\mathrm{a}+\mathrm{b}+)$ de negros nem com vários eritrócitos deste fenótipo em brancos.

Em 1982, Palatinik et a ${ }^{58}$ descreveram o anticorpo antiFs, que reagia preferencialmente com células Fy(a-b-), em uma mulata brasileira com fenótipo $\mathrm{Fy}(\mathrm{a}+\mathrm{b}+)$ e foi outro caso de um único exemplar detectado. Em 1987, Nichols et al ${ }^{15}$ descreveram o primeiro anticorpo monoclonal, que definiu o novo epítopo Fy6 e foi produzido por imunização de rato com um conjunto de eritrócitos humanos.

Outros anticorpos anti-Fy6 monoclonais foram produzidos e usados como instrumentos que auxiliaram tanto no isolamento da proteína Duffy, quanto para a clonagem desse gene. ${ }^{19,27,59,60}$

Para fins transfusionais é recomendado que, quando anticorpos Duffy forem identificados em amostras de sangue de pacientes, ou ainda naqueles que, apesar de não mais os demonstrarem no soro tiverem histórico dos mesmos, seja selecionado sangue antígeno-negativo. Em casos de anti-Fy3 e anti-Fy5, deve-se selecionar sangue com fenótipo Fy(a-b-) e para esse último pode-se também selecionar sangue $\mathrm{Rh}_{\text {null }}$ para transfusão. ${ }^{53}$

A importância do estudo por genotipagem de indivíduos com fenótipo Fy(a-b-) se faz necessária em pacientes com anemia falciforme ou cronicamente transfundidos, para melhor seleção de unidades de sangue a serem utilizadas, a fim de propiciar otimização do uso de unidades Fy(a-b-). ${ }^{61}$

\section{Doença hemolítica do recém-nascido}

Tanto anti-Fy $\mathrm{y}^{\mathrm{a}}$ como anti-Fy ${ }^{\mathrm{b}}$ podem causar doença hemolítica do recém-nascido (DHRN), geralmente não levam à morbidade significante e não são freqüentes. Entretanto, há relatos em que foram necessárias transfusões intra-útero e ex-sangüíneo transfusão. ${ }^{62-66}$

Os antígenos Duffy foram detectados em eritrócitos de fetos entre 6 e 7 semanas de vida e estão bem desenvolvidos ao nascimento. Parece que a quantidade desses antígenos não varia durante a vida. ${ }^{56,57}$

O primeiro caso descrito de transfusão intra-útero devido à incompatibilidade materno-fetal por anti-Fya foi descrito por Cook et al, ${ }^{68} \mathrm{em} 1984$, mas, após o nascimento, esse recém-nascido não necessitou de transfusão de concentrado de hemácias, apresentando somente um teste de antiglobulina direto fracamente positivo. Em estudo realizado por Geifman-Holtzman et al, ${ }^{69} \mathrm{em} 452$ mulheres em idade reprodutiva, encontraram anti-Fy em 5,4\% e anti-Fy ${ }^{\mathrm{b}}$ em $0,2 \%$.

É possível a detecção de fetos em risco de desenvolver DHRN pela determinação do genótipo Duffy em líquido amniótico. ${ }^{70}$

\section{Gene}

Historicamente, o gene Duffy foi o primeiro grupo sangüíneo cujo locus genético foi referido a um autossomo específico, o cromossomo 1 , e está localizado próximo à região centromérica. ${ }^{71}$ Inicialmente o locus $F Y$ foi localizado na região 1q21-25 por análise de ligação. ${ }^{72}$

Posteriormente, o cDNA correspondente ao mRNA da proteína Duffy foi clonado a partir uma biblioteca de medula óssea e seqüenciada ${ }^{19}$ e seu gene foi mapeado em 1q22-q23. ${ }^{73}$

Acreditava-se que esse gene consistia de apenas 1 exon, revelando uma estrutura primária de um polipeptídeo altamente hidrofóbico, contendo uma seqüência nonapeptídica N-terminal (MASSGYVLQ) composta de 338 aminoácidos chamado de transcrito menor. ${ }^{23,74}$ Um longo estudo subseqüente revelou a presença de um outro exon que contém seqüências codificadoras não traduzidas. Esse exon pode ser unido (após splicing) ao segundo exon (exon 2) para dar origem a 
um transcrito de 336 aminoácidos, contendo uma seqüência de um heptapeptídeo N-terminal (MGNCLHR) chamado de transcrito maior. ${ }^{75}$ Iwamoto, nesse estudo, determinou os níveis relativos da expressão dos $2 F Y$ mRNAs distintos verificando uma predominância do transcrito maior nas células eritróides e em todos os órgãos estudados.

Desde a descoberta, em 1995, por Iwamoto et al, ${ }^{25}$ que o gene $F Y$ é constituído de dois exons, os nucleotídeos são numerados utilizando o mRNA spliced; assim, o primeiro nucleotídeo do códon de iniciação da tradução (AUG) é o nucleotídeo $n^{0} 1{ }^{76}$ Numerando-se dessa forma, evitam-se as inconsistências criadas por diferentes tamanhos de $5^{\prime}$-UT aos diferentes sítios de iniciação da transcrição como descrito por Tournamille et $\mathrm{al}^{34} \mathrm{e}$ Iwamoto et al. ${ }^{75}$ Em nível protéico, a Metionina é numerada como $\mathrm{n}^{\circ} 1$.

\section{Estrutura da proteína}

Moore et al ${ }^{77}$ mostraram que, quando hemácias Fy $(\mathrm{a}+\mathrm{b}-)$ com superfície radio-iodo marcadas eram incubadas com anti-Fy antes da solubilização em detergente, uma proteína de 35 a $43 \mathrm{kD}$ era especificamente imunoprecipitada.

Hadley et al, ${ }^{78}$ utilizando técnica de immunoblotting com um soro potente anti-Fy ${ }^{\mathrm{a}}$, identificaram uma proteína com aparente massa molecular de 35-43 kDa.

Nichols et al, ${ }^{15}$ utilizando o soro monoclonal murino anti-Fy6, observaram que este reage com uma proteína de banda larga de 36-46 kDa em immunoblots de proteínas de membrana eritrocitárias detergente-solúveis.

Finalmente, um estudo por desialização da membrana do eritrócito por neuraminidase resultou numa alteração da mobilidade eletroforética da proteína Fy ${ }^{\mathrm{a}}$ de $35 \mathrm{kD}$ para 43 $\mathrm{kD}$, sugerindo que a proteína Duffy é uma glicoproteína. ${ }^{79}$

Chaudhuri et a ${ }^{18}$ mostraram que a proteína Duffy é $\mathrm{N}$-glicosilada, e a variação do grau de $\mathrm{N}$-glicosilação provavelmente contribui para a faixa de peso molecular de 35 a 45 $\mathrm{kDa}$, também chamada de GPD (glicoproteína Duffy). Embora esse estudo tenha deduzido que a seqüência de aminoácidos dos antígenos Duffy era uma proteína de nove passagens transmembrânicas, Neote et $\mathrm{al}^{74}$ mostraram a presença de sete hélices hidrofóbicas, sugerindo uma topologia na qual existem um domínio amino-terminal extracelular, três alças extracelulares, três alças citoplasmáticas e um domínio coboxiterminal citoplasmático, demonstrando que a glicoproteína Duffy era uma proteína de sete passagens transmembrânicas. O domínio extracelular N-terminal da proteína Duffy compreende 60 aminoácidos, as três alças extracelulares compreendem os resíduos de aminoácidos 119-129, 189-206 e 267-288.

Utilizando soros humanos anti-Fy ${ }^{\mathrm{a}}$, anti-Fy ${ }^{\mathrm{b}}$ e anti-IgG ferritina-marcada, Masouredis et a ${ }^{80}$ estimaram que hemácias Fy(a+b-) e Fy(a-b+) carreiam entre 13.000 e 14.000 sítios antigênicos $\mathrm{Fy}^{\mathrm{a}}$ e $\mathrm{Fy}^{\mathrm{b}}$ respectivamente. Como esperado, hemácias $\mathrm{Fy}(\mathrm{a}+\mathrm{b}+)$ têm a metade do número de sítios $\mathrm{Fy}^{\mathrm{a}}$ que nas hemácias de fenótipo $\mathrm{Fy}(\mathrm{a}+\mathrm{b}-)$.

\section{Expressão nos tecidos}

A glicoproteína Duffy é expressa em diversos tecidos não eritróides como o rim, baço, coração, pulmão, músculo, duodeno, pâncreas, placenta, cérebro, intestino, glândula tireóide e em células de Purkinje do cerebelo. ${ }^{23,48,81}$

As células responsáveis pela expressão de Duffy nesses tecidos são as células endoteliais que revestem as vênulas pós-capilares, exceto no cérebro, onde a expressão Duffy está localizada nas células de Purkinje. ${ }^{82,83,94}$

\section{Análise função-estrutura dos antígenos Duffy}

A discussão sobre a função dos antígenos eritrocitários Duffy iniciou-se em 1975 quando Miller et a ${ }^{16}$ sugeriram que os determinantes antigênicos $\mathrm{Fy}^{\mathrm{a}}$ ou $\mathrm{Fy}^{\mathrm{b}}$ poderiam ser receptores para Plasmodium knowlesi e que a resistência à invasão do merozoíto em negros devia-se ao fenótipo Duffy negativo. ${ }^{85}$

Para um melhor entendimento do envolvimento dos antígenos Duffy na fisiopatologia do Plasmodium, iremos brevemente discorrer sobre o ciclo de vida da malária.

A malária é causada pelo protozoário do gênero Plasmodium, e as espécies que afetam o ser humano são o Plasmodium vivax, Plasmodium falciparum, Plasmodium malariae, Plasmodium ovale. No caso brasileiro, destacamse as três primeiras espécies. O plasmódio infecta alternadamente um hospedeiro vertebrado e invertebrado. O vetor é a fêmea do mosquito Anopheles.

A fêmea do Anopheles se alimenta com sangue infectado com gametócitos promovendo o processo de fertilização, produzindo zigoto que se transforma em uma forma invasiva que cresce e se divide, produzindo milhares de esporozoítas invasivos, migrando pelo corpo e invadindo as glândulas salivares. A fêmea, ao se alimentar, inocula os esporozítos no sangue do homem, que migrarão rapidamente para os hepatócitos transformando-se em trofozoítos hepáticos onde amadurecem e dividem-se formando milhares de merozoítas. Os hepatócitos se rompem liberando os merozoítas na circulação, iniciando o ciclo eritrocítico. Essa parte do ciclo de vida não produz sintomas no hospedeiro. No ciclo eritrocitário, os merozoítas desenvolvem-se em trofozoítas formando os esquizontes. Estes, maduros, levam à ruptura da hemácia, que libera merozoítos capazes de reinfectar novas hemácias. Esse estágio do ciclo está associado com sintomas clínicos. Após um período de replicação assexuada, alguns merozoítos se diferenciam em gametócitos tornandose infectantes aos mosquitos. ${ }^{86}$

No Brasil, cabe ressaltar a elevada prevalência da malária, principalmente na região da Amazônia legal, que envolve os estados do Acre, Amazonas, Amapá, Maranhão, Mato Grosso, Pará, Roraima, Rondônia e Tocantins. Foram registrados, no ano de 2002, 241.806 casos de malária, e, em 2003, 208.718 novos casos. 
O mecanismo de entrada no eritrócito pelo Plasmodium foi estudado por Aikawa et al, ${ }^{88}$ que observaram, por microscopia eletrônica, que a porção apical do merozoíto faz o contato inicial com o eritrócito, criando uma pequena depressão na membrana. Essa área começa a espessar e forma uma junção com a membrana do merozoíto. Então, o merozoíta entra na superfície do eritrócito por invaginação. Após a entrada completa do Plasmodium, o orifício de entrada fechase atrás dele. Essa junção tem importância crucial para a invasão do parasita. ${ }^{89}$

As pesquisas sobre citocinas e seus receptores convergiram na investigação dos antígenos de grupo sangüíneo Duffy, mostrando uma importante função fisiológica para os alelos dessa glicoproteína.

As quimiocinas (citocinas com função de quimiotaxia) são parte de um grande membro da família de citocinas, estruturalmente homólogas. As quimiocinas são divididas em duas grandes subfamílias e se diferenciam por dois resíduos cisteína de aminoácidos terminais que participam na ligação dissulfeto. Podem estar adjacentes (quimiocinas $\mathrm{C}-\mathrm{C}$ ) ou separados por um aminoácido (quimiocinas $\mathrm{C}-\mathrm{X}-\mathrm{C}$ ). As quimiocinas dessas subfamilias são produzidas por leucócitos e por vários tipos de células teciduais como as endoteliais, epiteliais e fibroblastos. ${ }^{20,90}$

$\mathrm{Na}$ inflamação aguda, as quimiocinas C-X-C atuam principalmente sobre os neutrófilos, e, na inflamação crônica, as quimiocinas $\mathrm{C}-\mathrm{C}$ atuam principalmente sobre monócitos, linfócitos e eosinófilos. ${ }^{91}$

Cada quimiocina tem um receptor específico no qual se liga com alta afinidade. Em 1991, Darbonne et a ${ }^{92}$ descobriram um novo receptor de quimiocinas nos eritrócitos. Revisando a topografia dos receptores de quimiocinas e analisando o repertório conhecido de ligantes para essa nova descoberta, verificaram que esse novo receptor eritróide era um ligante da família C-X-C com alta afinidade para MGSA e, principalmente, para Interleucina-8 (IL-8), e C-C com alta afinidade para MCP-1 (Proteína Quimotática de Monócito), MIP-1 (Proteína Inflamatória de Macrófago 1) e RANTES (Células Expressas e Secretadas Reguladas na Ativação). ${ }^{21,93.94}$

Horuk et a ${ }^{95}$ mostraram, em um estudo sobre receptores de quimiocinas multiespecíficos em eritrócitos humanos, que a IL-8 liga-se minimamente a eritrócitos Duffy negativos, que anticorpos monoclonais para antígenos Duffy bloqueavam a ligação de IL-8 em eritrócitos Duffy positivo, e que a IL-8 também impedia a ligação e a invasão por $P$. knowlesi em hemácias, levando a diversas linhas de evidências, indicando que os antígenos de grupo sangüíneo Duffy são receptores de quimiocinas.

Outros estudos estabeleceram a relação entre receptores de quimiocinas nos eritrócitos humanos e antígenos Duffy, por alinhamento da seqüência da proteína Duffy com outros membros da família de quimiocinas.

Foi visto também que a organização dos dois exons $F Y$ é a mesma encontrada nos genes de outros receptores de quimiocinas. A partir de então, os antígenos eritrocitários Duffy foram denominados DARC (Duffy Antigen Receptor Chemokines). ${ }^{74,96,97}$

A IL-8 é o ligante do DARC eritróide mais intensivamente estudado, é de baixo peso molecular, com aproximadamente $8 \mathrm{kDa}$, e é produzida pela maioria das células do organismo, particularmente por macrófagos e células endoteliais, sendo envolvida na migração celular. A IL-8 é conhecida por ser um potente indutor de quimiotaxia para neutrófilos. ${ }^{98}$ Após sua introdução na circulação, a IL-8 é rápida e eficientemente ligada ao DARC eritróide, ficando incapaz de ativar neutrófilos e, desse modo, o DARC eritróide pode funcionar como um "escoadouro" para a IL-8 lançada na circulação.99,100

Assim, a absorção de IL-8 nos eritrócitos pelo DARC pode funcionar como uma limitante da estimulação de leucócitos pela diminuição da IL-8 sangüínea. ${ }^{92}$

A ausência do DARC eritróide em indivíduos Duffy negativo conseqüentemente reduz a capacidade de ligação da IL-8 aos eritrócitos. ${ }^{95}$

O significado biológico do DARC nos eritrócitos inicialmente pareceu questionável, devido à falta de associação entre doenças e fenótipo Duffy negativo. ${ }^{76}$ Porém, a relação função-estrutura e a localização do DARC na fisiologia normal e patológica ainda permanecem um campo vasto para novas descobertas.

A localização do DARC nas células endoteliais das vênulas pós-capilares aliada à sua capacidade de fixar as quimiocinas sugere que essa proteína pode ter um papel na cascata inflamatória, participando na formação do gradiente de quimiocinas que permite lançar os linfócitos na circulação para os sítios de inflamação. ${ }^{101}$ Estudos realizados por Middleton et al ${ }^{102}$ mostraram que o DARC estava implicado na transcitose e na apresentação de IL-8 produzidas pelas células epiteliais para a superfície luminal das células endoteliais das vênulas pós-capilares.

Recente artigo mostra que os antígenos Duffy facilitam a movimentação de quimiocinas através do endotélio in vitro e promove a transmigração de neutrófilos in vivo e in vitro. ${ }^{103}$

A expressão aumentada do RNAm da proteína Duffy durante condições inflamatórias no rim sugere que uma das propriedades dos antígenos Duffy como ligantes de quimiocinas seletivos são biologicamente relevantes durante processos inflamatórios. ${ }^{104,105}$

Segerer et al ${ }^{106}$ observaram o aumento da expressão de DARC em pacientes que apresentavam rejeição celular e humoral de transplante renal associado à maior deposição da fração C4d em capilares peritubulares.

A diminuição da função renal após transplante está mais associada a pacientes com fenótipo Fy(a-b-), que pode ser devido a ausência de receptores DARC, que faz o papel de atenuar os efeitos inflamatórios, agindo como um escoadouro de quimiocinas. ${ }^{107}$

Foi visto que a expressão dos antígenos Duffy estava aumentada nos leitos vasculares e nos alvéolos pulmonares 
durante o quadro de pneumonia supurativa, sugerindo que os antígenos Duffy apresentavam um papel funcional no parênquima pulmonar durante o processo inflamatório. ${ }^{108}$

Foi demonstrado também que os receptores DARC são receptores inflamatórios seletivos e não homeostáticos, fornecendo evidências que as quimiocinas ligantes ao antígeno Duffy são padrão em células endoteliais de tecidos inflamados e não em tecidos íntegros. ${ }^{109}$

Um estudo realizado por Lachgar et al ${ }^{110}$ mostrou que o vírus HIV-1 se liga aos eritrócitos dos indivíduos caucasianos através do DARC, fazendo os eritrócitos capazes de transmitir o HIV às células mononucleares do sangue periférico, sugerindo que os eritrócitos podem funcionar como um reservatório para HIV-1 ou como um receptor para a entrada de HIV-1 em células CD4+ bem como em neurônios ou células endoteliais.

Além das patologias descritas anteriormente, outros artigos demonstram que os receptores DARC estão envolvidos na angiogênese do câncer e para isso iremos relatar brevemente os principais eventos relacionados a esse processo.

A angiogênese é essencial em diversos processos fisiológicos, como a embriogênese, reparação de ferimentos e ciclo menstrual, e processos patológicos, como o crescimento tumoral e metástase.

A angiogênese tumoral tem por característica a rápida resposta do sistema vascular ao aumento da necessidade metabólica do tecido tumoral, aumentando a microvascularização e o recrutamento de células endoteliais progenitoras circulantes, sendo esse processo muito controlado devido ao alto custo metabólico da angiogênese, ocorrendo somente quando for necessário. No caso do tumor, esse controle estrito é perdido, levando a uma angiogênese aberrante. ${ }^{111,112}$

Em cada uma das etapas da angiogênese, os mecanismos exatos e os mediadores específicos que orquestram estes eventos permanecem interrogados. Entretanto, quimiocinas CXC, como a IL-8, demonstraram ter um papel crescente neste processo. ${ }^{111,113}$

A incidência do câncer de próstata em afro-americanos é $60 \%$ maior do que aquela encontrada nos caucasianos, com uma taxa de mortalidade duas vezes mais elevada. Dada a importância das quimiocinas angiogênicas no desenvolvimento da rede vascular tumoral e o DARC ter propriedades ligantes com alta afinidade à IL-8, a possibilidade de homens afro-americanos com ausência do DARC eritróide pode ser considerado um fator de predisposição genético para maior incidência e mortalidade para o câncer de próstata. ${ }^{14,115}$

Finalmente, pode-se dizer que, nos últimos dez anos, os progressos revelados pelas pesquisas conduzidas tanto no Brasil como internacionalmente tiveram forte impacto para melhor entendimento desse sistema de grupo sangüíneo, embora muito há que se desvendar, em especial quanto à função biológica dos antígenos do sistema Duffy.

\section{Abstract}

After the introduction of the indirect antiglobulin technique by Coombs in the middle of the 1940's, several antibodies have been discovered. Duffy blood group system came to light when Cutbush and Ikin detected the first antibodies related to this system in the beginning of the 1950's. The antibodies of this system are clinically significant in transfusional practice as they have been involved in hemolytic transfusion reactions and hemolytic disease of the newborn, showing them to be of worldwide occurrence. The FY gene is constituted of two exons and its locus was mapped on chromosome 1q22-q23. The $F y^{a}$ and $F y^{b}$ antigens are encoded by FYA and FYB alleles, and are responsible for the $F y(a+b-), F y(a-b+)$ and $F y(a+b+)$ phenotypes. They are carried by a 336 amino acid glycoprotein called DARC (Duffy Antigen/Receptor for Chemokines) which has high affinity to chemokines, also being Plasmodium vivax receptors. The polymorphisms related to its alleles have led to the development of a PCR genotyping technique, which is useful for the safety of blood transfusion, and determining fetus-maternal incompatibilities. In the last decade, much research has been done to determine the biological role of blood group antigens. In this paper we reviewed the Duffy Blood Group System, especially in respect to transfusional practice and biological functions. Rev. bras. hematol. hemoter. 2005;27(2):110-119.

Key words: Duffy blood group system; DARC; chemokines; malaria; DUFFY gene.

\section{Referências Bibliográficas}

1. Landsteiner K. Individual differences in human blood. Science 1931;73:405.

2. Levine P, Burnham L, Katzin EM, Vogel P. Role of isoimmunization in pathogenesis of erythroblastosis fetalis. Am J Obstet Gynecol 1941;42:925.

3. Storry JR, Olsson ML. Genetic basis of blood group diversity. Br J Haematol 2004;126(6):759-71. Review.

4. Hamblin MT, Di Rienzo A. Detection of the signature of natural selection in humans: evidence from the Duffy blood group locus. Am J Hum Genet 2000;66(5):1669-79. Erratum in: Am J Hum Genet 2002;70(1):284.

5. Hamblin MT, Thompson EE, Di Rienzo A. Complex signatures of natural selection at the Duffy blood group locus. Am J Hum Genet. 2002;70(2):369-83.

6. Cutbush M, Mollison PL, Parkin DM. A new human blood group. Nature 1950;165-88.

7. Cutbush M, Mollison PL. The Duffy blood group system. Heredity 1950;4:383-89.

8. Ikin EW, Mourant AE, Pettenkoffer HJ, Blumenthal G. Discovery of the excepted haemagglutinin anti-Fyb. Nature 1951;168:1.077.

9. Sanger R, Race RR, Jack J. The Duffy blood groups of New York negroes: the phenotype Fy (a-b-). Br J Haematol 1955;1(4):370-4.

10. Chown B, Lewis M, Kaita H. The Duffy blood group system in Caucasians: evidence for a new allele. American Journal of Human Genetics 1965;17:384-9.

11. Lewis M, Kaita H, Chown B. The Duffy blood group system in Caucasians. A further population sample. Vox Sang 1972;23:523-7.

12. Albrey JA, Vincent EE, Hutchinson J, et al. A new antibody, anti-Fy3, in the Duffy blood group system. Vox Sang 1971 Jan;20(1):29-35.

13. Behzad O, Lee CL, Gavin J, Marsh WL. A new anti-erythrocyte antibody in the Duffy system: anti-Fy4. Vox Sang 1973;24:337-42. 
14. Colledge KI, Pezzulich M, Marsh WL. Anti-Fy5, and antibody disclosing a probable association between the Rhesus and Duffy blood group genes. Vox Sang 1973 Mar; 24(3):193-9.

15. Nichols ME, Rubinstein P, Barnwell J, et al. A new human Duffy blood group specificity defined by a murine monoclonal antibody. Immunogenetics and association with susceptibility to Plasmodium vivax. J Exp Med 1987 1;166(3):776-85.

16. Miller LH, Mason SJ, Dvorak JA, et al Erythrocyte receptors for (Plasmodium knowlesi) malaria: Duffy blood group determinants. Science 1975; 189:561-3

17. Barnwell JW, Nichols ME, Rubinstein P. In vitro evaluation of the role of the Duffy blood group in erythrocyte invasion by Plasmodium vivax. J Exp Med 1989 1;169(5):1795-802.

18. Chaudhuri A, Zbrzezna V, Johnson C, et al. Purification and characterization of an erythrocyte membrane protein complex carrying Duffy blood group antigenicity. Possible receptor for Plasmodium vivax and Plasmodium knowlesi malaria parasite. J Biol Chem 1989 15;264(23):13770-4.

19. Chaudhuri A, Polyakova J, Zbrzezna V, et al. Cloning of glycoprotein D cDNA, which encodes the major subunit of the Duffy blood group system and the receptor for the Plasmodium vivax malaria parasite. Proc Natl Acad Sci 1993 15;90 (22):10793-7.

20. Rollins BJ. Chemokines. Blood 1997 1;90(3):909-28. Review.

21. Horuk R, Colby TJ, Darbonne WC, et al. The human erythrocyte inflammatory peptide (chemokine) receptor. Biochemical characterization, solubilization, and development of a binding assay for the soluble receptor. Biochemistry 1993 8;32(22):5733-78.

22. http://www.iccbba.com/wpantigentables.htm (acesso em 07. 01. 2005)

23. Chaudhuri A, Polyakova J, Zbrzezna V, Pogo AO. The coding sequence of Duffy blood group gene in humans and simians: restriction fragment length polymorphism, antibody and malarial parasite specificities, and expression in non-erythroid tissues in Duffy-negative individuals. Blood 1995;85:615-21.

24. Mallinson G, Soo KS, Schall TJ, et al. Mutations in the erythrocyte chemokine receptor (Duffy) gene: the molecular basis of the Fya/ $\mathrm{Fy}^{\mathrm{b}}$ antigens and identification of a deletion in the Duffy gene of an apparently healthy individual with the Fy(a-b-) phenotype. Br J Haematol 1995;90(4):823-9.

25. Iwamoto S, Omi T, Kajii E, Ikemoto S. Genomic organization of the glycoprotein D gene: Duffy blood group $\mathrm{Fy}^{\mathrm{a}} / \mathrm{Fy}^{\mathrm{b}}$ alloantigen system is associated with a polymorphism at the 44-amino acid residue. Blood 1995 1;85(3):622-6.

26. Tournamille C, Le Van Kim C, Gane P, et al. Molecular basis and PCR-DNA typing of the Fya/Fyb blood group polymorphism. Hum Genet 1995;95:407-10.

27. Wasniowska K, Lisowska E, Halverson GR, et al. The Fy', Fy6 and Fy3 epitopes of the Duffy blood group system recognized by new monoclonal antibodies: identification of a linear Fy3 epitope. $\mathrm{Br}$ J Haematol 2004;124(1):118-22.

28. Tournamille C, Le Van Kim C, Gane P, et al. Arg89Cys substitution results in very low membrane expression of the Duffy antigen/ receptor for chemokines in $\mathrm{Fy}(\mathrm{x})$ individuals. Blood 1998;92 (6):2.147-56

29. Olsson ML, Smythe JS, Hansson C, et al. The Fy(x) phenotype is associated with a missense mutation in the $\mathrm{Fy}(\mathrm{b})$ allele predicting Arg89Cys in the Duffy glycoprotein. Br J Haematol 1998;103 (4):1184-91.

30. Cedergren B, Giles CM. An FyxFyx individual found in northern Sweden. Vox Sang 1973;24:264-6.

31. Buchanan DE, Sinclair M, Sanger R, et al. An Alberta ree Indian with a rare Duffy antibody anti-Fy3. Vox Sang 1976;30:114-21.

32. Habibi B, Perrier P, Salmon C. HD50 assay evolution of the antigens Fy3 depression in Fyx individuals. J Immunogenet 1980;7:191-3.
33. Castilho L, Rios M, Pellegrino J Jr, et al. A novel FY allele in Brazilians. Vox Sang 2004;87(3):190-5.

34. Tournamille C, Colin Y, Cartron JP, Le Van Kim C. Disruption of a GATA motif in the Duffy gene promoter abolishes erythroid gene expression in Duffy-negative individuals. Nat Genet 1995; $10(2): 224-8$.

35. Iwamoto S, Li J, Sugimoto N, et al. Characterization of the Duffy gene promoter: evidence for tissue-specific abolishment of expression in Fy(a-b-) of black individuals. Biochem Biophys Res Commun 1996 24;222(3):852-9.

36. Le Pennec PY, Rouger P, Klein MT, et al. Study of anti-Fy in five black Fy(a-b-) patients. Vox Sang 1987; 52(3):246-9.

37. Yazdanbakhsh K, Rios M, Storry JR, et al. Molecular mechanisms that lead to reduced expression of Duffy antigens. Transfusion 2000 Mar;40(3):310-20.

38. Olsson ML, Hansson C, Avent ND, et al. A clinically applicable method for determining the three major alleles at the Duffy (FY) blood group locus using polymerase chain reaction with allelespecific primers. Transfusion 1998;38(2):168-73.

39. Zimmerman PA, Woolley I, Masinde GL, et al. Emergence of FY*A(null) in a Plasmodium vivax - endemic region of Papua New Guinea. Proc Natl Acad Sci USA 1999 23;96(24):13973-7.

40. Langhi Jr DM, Albuquerque S, Covas DT, et al. Presença do alelo FYAnull, do sistema de grupo sanguíneo Duffy em habitantes de região endêmica para malaria e doadores de sangue no Brasil. Rev Bras Hemat Hemot 2004;26(2):268-9.

41. Shimizu Y, Kimura M, Settheetham-Ishida W, et al. Genotyping of Duffy blood group in several Thai ethnic groups. Southeast Asian J Trop Med Public Health 1997;28(1):32-5.

42. Shimizu Y, Ao H, Soemantri A, et al. Sero- and molecular typing of Duffy blood group in Southeast Asians and Oceanians. Hum Biol 2000;72(3):511-8.

43. Lewis GE Jr, Miller LH, Ibrahim L, et al. Duffy phenotypes in Malaysian populations: correction of previous unusual findings. Trans R Soc Trop Med Hyg 1988;82 (3):509-10.

44. Rios M, Chaudhuri A, Mallinson G, et al. New genotypes in Fy(a-b-) individuals: nonsense mutations (Trp to stop) in the coding sequence of either FY A or FY B. Br J Haematol 2000; 108(2):448-54.

45. Reid ME, Lomas-Francis C. The blood group antigen. Facts book, 1st edn. 1997. California. Academic Press

46. Tournamille C, Le Van Kim C, Gane P, et al. Close association of the first and fourth extracellular domains of the Duffy antigen/ receptor for chemokines by a disulfide bond is required for ligand binding. J Biol Chem 1997 27;272(26):16274-80.

47. Judson PA, Anstee DJ. Comparative effect of trypsin and chymotrypsin on blood group antigens. Med Lab Sci 1977 Jan; 34(1):1-6

48. Hadley TJ, Peiper SC. From malaria to chemokine receptor: the emerging physiologic role of the Duffy blood group antigen. Blood 1997;89(9):3077-91.

49. Woolley IJ, Hotmire KA, Sramkoski RM, et al. Differential expression of the Duffy antigen receptor for chemokines according to RBC age and FY genotype. Transfusion 2000;40(8):949-53.

50. Novaretti MCZ, Dorlhiac-Llacer PE, Chamone DAF - Estudo de grupos sangüíneos em doadores de sangue caucasóides e negróides da cidade de São Paulo. Rev Bras Hemat Hemot 2000; 22(1):23-32.

51. Moulds JM, Hayes S, Wells TD. DNA analysis of Duffy genes in American blacks. Vox Sang 1998;74(4):248-52.

52. Sandler SG, Schiliro G, Russo A, et al. Blood group phenotypes and the origin of sickle cell hemoglobin in Sicilians. Acta Haematol 1978;60(6):350-7.

53. Daniels G, Poole J, de Silva M, et al. The clinical significance of blood group antibodies. Transfus Med 2002;12:287-95. 
54. Szymanski IO, Huff SR, Delsignore R. An autoanalyzer test to determine immunoglobulin class and IgG subclass of blood group antibodies. Transfusion 1982;22(2):90-5.

55. Mollinson PL, Engelfriet CP, Contreras M, editors. Blood Transfusion in Clinical Medicine. 1997 Great Britain, UK: Blackwell Science. Pg 191

56. Issitt PD, Anstee DJ, editors. Applied Blood Group Serology, 4 th edn. 1998 Durban, North Carolina USA: Montgomery Scientific Publications. Pg 440-1.

57. Kim HH, Park TS, Oh SH, et al. Delayed hemolytic transfusion reaction due to anti-Fy ${ }^{\mathrm{b}}$ caused by a primary immune response: a case study and a review of the literature. Immunohematol 2004; 20(3):184-6.

58. Palatinik M, Junqueira PC, Alves ZMS. Fs: an antigenic determinant possible related to the Duffy blood group. Rev Franc Transfus Immuno-Hémat 1982;25:629-37.

59. Riwom S, Janvier D, Navenot JM, et al. Production of a new murine monoclonal antibody with Fy6 specificity and characterization of the immunopurified N-glycosylated Duffy-active molecule. Vox Sang 1994;66(1):61-7.

60. Wasniowska K, Petit-LeRoux Y, Tournamille C, et al. Structural characterization of the epitope recognized by the new anti-Fy 6 monoclonal antibody NaM 185-2C3. Transfus Med 2002; 12(3): 205-11.

61. Rios M, Reid ME, Naime D, et al. Importance of GATA box analysis in genotyping for the Duffy blood group system. Transfusion 1997; 37(Suppl 9S):101S, Abstract.

62. Westhoff CM, Reid ME. Review: the Kell, Duffy, and Kidd blood group systems. Immunohematol 2004;20(1):37-49.

63. Goodrick MJ, Hadley AG, Poole G. Haemolytic disease of the fetus and newborn due to anti-Fy(a) and the potential clinical value of Duffy genotyping in pregnancies at risk. Transfus Med 1997; 7(4):301-4.

64. Weinstein L, Taylor ES. Hemolytic disease of the neonate secondary to anti-Fya. Am J Obstet Gynecol 1975 1;121 (5): 643-5.

65. Babinszki A, Berkowitz RL. Haemolytic disease of the newborn caused by anti-c, anti-E and anti-Fya antibodies: report of five cases. Prenat Diagn 1999;19(6):533-6.

66. Dufour P, Vinatier D, Bernardi C, et al. Severe fetomaternal antiDuffy allo-immunization. J Gynecol Obstet Biol Reprod (Paris). 1991;20(6):809-14. Review.

67. Toivanen P, Hirvonen T. Antigens Duffy, Kell, Kidd, Lutheran and Xg a on fetal red cells. Vox Sang 1973;24(4):372-6.

68. Cook SG, Baker JW, Weaver EW. Intrauterine transfusion for antiDuffy (Fy) haemolytic disease. Aust N Z J Obstet Gynaecol 1989; 29:263-4.

69. Geifman-Holtzman O, Wojtowycz M, Kosmas E, Artal R. Female alloimmunization with antibodies known to cause hemolytic disease. Obstet Gynecol 1997;89(2):272-5. Review.

70. Hessner MJ, Pircon RA, Johnson ST, Luhm RA. Prenatal genotyping of the Duffy blood group system by allele-specific polymerase chain reaction. Prenat Diagn 1999;19(1):41-5.

71. Donahue RP, Bias WB, Renwic JH, McKusick VA. Probable assignment of the Duffy blood group locus to chromosome 1 in man. Proc Nat Acad Sci 1968;61: 949-55.

72. Dracopoli NC, O'Connell P, Elsner TI, et al. The CEPH consortium linkage map of human chromosome 1. Genomics 1991;9(4):686700 .

73. Mathew S, Chaudhuri A, Murty VV, Pogo AO. Confirmation of Duffy blood group antigen locus (FY) at 1q22-->q23 by fluorescence in situ hybridization. Cytogenet Cell Genet 1994;67(1):68.

74. Neote K, Mak JY, Kolakowski LF Jr, Schall TJ. Functional and biochemical analysis of the cloned Duffy antigen: identity with the red blood cell chemokine receptor. Blood 1994;84:44-52.
75. Iwamoto $\mathrm{S}$, Li J, Omi T, et al. Identification of a novel exon and spliced form of Duffy mRNA that is the predominant transcript in both erythroid and postcapillary venule endothelium. Blood 1996 1;87(1):378-85.

76. Pogo AO, Chaudhuri A. The Duffy protein: a malarial and chemokine receptor. Semin Hematol 2000;37(2):122-9. Review.

77. Moore S, Woodrow CF, McClelland DBL. Isolation of membrane components associated with human red cell antigens Rho(D), (c), (E) and Fya. Nature 1982;295:529-31.

78. Hadley TJ, David PH, McGinnis MH, Miller LH. Identification of an erythrocyte component carrying the Duffy blood group antigens Fya antigen. Science 1984;223:597-9.

79. Tanner MJ, Anstee DJ, Mallinson G, et al. Effect of endoglycosidase F-peptidyl N-glycosidase F preparations on the surface components of the human erythrocyte. Carbohydr Res 1988 15;178:203-12.

80. Masouredis SP, Sudora E, Mahan L, Victoria EJ. Quantitative immunoferritin microscopy of Fya, Fyb, Jka, U, and Dib antigen site numbers on human red cells. Blood 1980;56(6):969-77.

81. Hesselgesser J, Horuk R. Chemokine and chemokine receptor expression in the central nervous system. J Neurovirol 1999; 5(1):13-26.

82. Hadley TJ, Lu ZH, Wasniowska K, et al. Postcapillary venule endothelial cells in kidney express a multispecific chemokine receptor that is structurally and functionally identical to the erythroid isoform, which is the Duffy blood group antigen. J Clin Invest 1994;94(3):985-91.

83. Peiper SC, Wang ZX, Neote K, et al. The Duffy antigen/receptor for chemokines (DARC) is expressed in endothelial cells of Duffy negative individuals who lack the erythrocyte receptor. J Exp Med 1995 1;181(4):1311-7.

84. Chaudhuri A, Nielsen S, Elkjaer ML, et al. Detection of Duffy antigen in the plasma membranes and caveolae of vascular endothelial and epithelial cells of nonerythroid organs. Blood 1997 15;89(2):701-12.

85. Miller LH, Mason SJ, Clyde DF, McGinniss MH. The resistance factor to Plasmodium vivax in blacks. The Duffy-blood-group genotype, FyFy.N Engl J Med 1976;295(6):302-4.

86. Ferreira AW, Ávila SLM. Diagnóstico Laboratorial. Guanabara Koogan $20012^{\mathrm{a}}$ edição. Cap 25: 263-73.

87.http://portal.saude.gov.br/saude/arquivos/pdf/be_malaria_01_2003. pdf (Acesso em 16 de novembro de 2004).

88. Aikawa M, Miller LH, Johnson J, Rabbege J. Erythrocyte entry by malarial parasites. A moving junction between erythrocyte and parasite. J Cell Biol 1978;77(1):72-82.

89. Miller LH, Aikawa M, Johnson JG, Shiroishi T. Interaction between cytochalasin B-treated malarial parasites and erythrocytes. Attachment and junction formation. J Exp Med 1979;149(1):172-84.

90. Walz W, Calandra T, Koth M, KotbM. Cytokines and Chemokines in Infectious Diseases Handbook. Humana Press 2003. Chap $11: 248-9$

91. Abbas AK, Lichtman AH, Pober JS. Imunologia Celular e Molecular. Revinter 2003 4a edição. Cap 11: 248.

92. Darbonne WC, Rice GC, Mohler MA, et al. Red blood cells are a sink for interleukin 8, a leukocyte chemotaxin. J Clin Invest 1991;88(4):1362-9.

93. Dohlman HG, Thorner J, Caron MG, Lefkowitz RJ. Model systems for the study of seven-transmembrane-segment receptors. Annu Rev Biochem 1991;60:653-88.

94. Neote K, Darbonne W, Ogez J, et al. Identification of a promiscuous inflammatory peptide receptor on the surface of red blood cells. J Biol Chem 1993 15;268(17):12247-9.

95. Horuk R, Chitnis CE, Darbonne WC, et al. A receptor for the malarial parasite Plasmodium vivax: the erythrocyte chemokine receptor. Science 1993;261:1182-4. 
96. Chaudhuri A, Zbrzezna V, Polyakova J, et al. Expression of the Duffy antigen in K562 cells. Evidence that it is the human erythrocyte chemokine receptor. J Biol Chem 1994; 269: $7835-8$.

97. Murphy PM. The molecular biology of leukocyte chemoattractant receptors. Annu Rev Immunol. 1994;12:593-633.

98. Roitt I, Brostoff J, Male D. Imunologia. Manole Ltda $19974^{\mathrm{a}}$ edição. Cap 8.11.

99. Van der Laken CJ, Boerman OC, Oyen WJ, et al. The kinetics of radiolabelled interleukin-8 in infection and sterile inflammation. Nucl Med Commun 1998;19(3):271-81.

100. de Winter RJ, Manten A, de Jong YP, et al. Interleukin 8 released after acute myocardial infarction is mainly bound to erythrocytes Heart 1997;78(6):598-602.

101. Tournamille C. Bases moléculaires et relations struture-fonction des antigènes de groupe sanguin Duffy: récepteur de chimiokines et de Plasmodium vivax. Transfus Clin Biol 2000;7:497-509

102. Middleton J, Neil S, Wintle J, et al. Transcytosis and surface presentation of IL-8 by venular endothelial cells. Cell 1997 31;91(3):385-95

103. Lee JS, Frevert CW, Wurfel MM, et al. Duffy antigen facilitates movement of chemokine across the endothelium in vitro and promotes neutrophil transmigration in vitro and in vivo. $\mathrm{J}$ Immunol 2003 15;170(10):5244-51.

104. Liu XH, Hadley TJ, Xu L, et al. Up-regulation of Duffy antigen receptor expression in children with renal disease. Kidney Int 1999;55(4):1491-500.

105. Segerer S, Regele H, MacK M, et al. The Duffy antigen receptor for chemokines is up-regulated during acute renal transplant rejection and crescentic glomerulonephritis. Kidney Int 2000;58 (4):1546-56

106. Segerer S, Bohmig GA, Exner M, et al. When renal allografts turn DARC. Transplantation 2003 15;75(7): 1030-4.

107. Akalin E, Neylan JF. The influence of Duffy blood group on renal allograft outcome in African Americans. Transplantation 2003 $15 ; 75(9): 1496-500$.
108. Lee JS, Frevert CW, Thorning DR, et al. Enhanced expression of Duffy antigen in the lungs during suppurative pneumonia. J Histochem Cytochem 2003;51(2):159-66.

109. Gardner L, Patterson AM, Ashton BA, et al. The human Duffy antigen binds selected inflammatory but not homeostatic chemokines. Biochem Biophys Res Commun. 2004 20;321(2) : 306-12.

110. Lachgar A, Jaureguiberry G, Le Buenac H, et al. Binding of HIV1 to RBCs involves the Duffy antigen receptors for chemokines (DARC). Biomed Pharmacother 1998;52(10):436-9.

111. Strieter RM, Belperio JA, Phillips RJ, Keane MP. CXC chemokines in angiogenesis of cancer. Semin Cancer Biol 2004; 14(3):195-200.

112. Moldovan NI. Tissular insemination of progenitor endothelial cells: the problem, and a suggested solution. Adv Exp Med Biol. 2003;522:99-113.

113. Li A, Dubey S, Varney ML, et al. IL-8 directly enhanced endothelial cell survival, proliferation, and matrix metalloproteinases production and regulated angiogenesis. J Immunol 2003 15; 170(6):3369-76.

114. Moore BB, Arenberg DA, Stoy K, et al. Distinct CXC chemokines mediate tumorigenicity of prostate cancer cells. Am J Pathol 1999; 154:1503-12.

115. Lentsch AB. The Duffy antigen/receptor for chemokines (DARC) and prostate cancer. A role as clear as black and white? Faseb J. 2002;16(9):1093-5

Avaliação: Editor e dois revisores externos Conflito de interesse: não declarado

Recebido: 03/03/05

Aceito: $28 / 03 / 05$ 